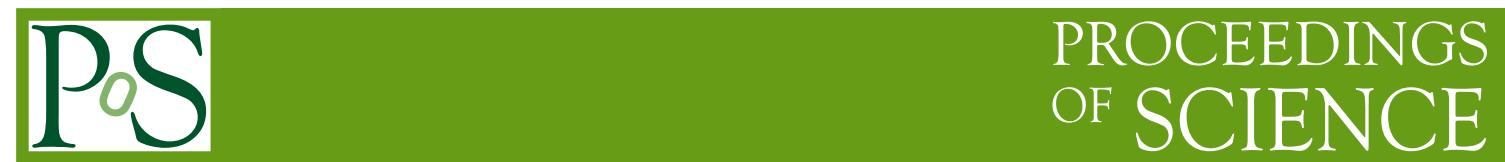

\title{
Forward physics with CMS
}

\author{
Monika GROTHE* \\ University of Wisconsin, Madison, USA \\ E-mail: monika.grothe@cern.ch \\ On behalf of the CMS collaboration
}

\begin{abstract}
Forward physics with CMS at the LHC covers a wide range of physics subjects, including very low- $x_{B j}$ QCD, underlying event and multiple interactions characteristics, $\gamma$-mediated processes, shower development at the energy scale of primary cosmic ray interactions with the atmosphere, diffraction in the presence of a hard scale and even MSSM Higgs discovery in central exclusive production. Selected feasibility studies to illustrate the forward physics potential of CMS are presented.
\end{abstract}

2008 Physics at LHC

September 29 - 4 October 2008

Split, Croatia

${ }^{*}$ Speaker. 


\section{Introduction}

Forward physics at the LHC covers a wide range of diverse physics subjects that have in common that particles produced at small polar angles, $\theta$, and hence large values of rapidity provide a defining characteristics. At the Large-Hadron-Collider (LHC), where proton-proton collisions occur at center-of-mass energies of $14 \mathrm{TeV}$, the maximal possible rapidity is $y_{\text {max }}=\ln \frac{\sqrt{s}}{m_{\pi}} \sim 11.5$. The central components of CMS are optimized for efficient detection of processes with large polar angles and hence high transverse momentum, $p_{T}$. They extend down to about $|\theta|=1^{\circ}$ from the beam axis or $|\eta|=5$, where $\eta=-\ln [\tan (\theta / 2)]$ is the pseudorapidity. In the forward region, the central CMS components are complemented by several CMS and TOTEM subdetectors with coverage beyond $|\eta|=5$. TOTEM is an approved experiment at the LHC for precision measurements of the $p p$ elastic and total cross sections. The combined CMS and TOTEM apparatus comprises two suites of calorimeters with tracking detectors in front plus near-beam proton taggers. The CMS Hadron Forward (HF) calorimeter with the TOTEM telescope T1 in front covers the region $3<|\eta|<5$, the CMS CASTOR calorimeter with the TOTEM telescope T2 in front covers $5.2<|\eta|<6.6$. The CMS ZDC calorimeters are installed at the end of the straight LHC beam-line section, at a distance of $\pm 140 \mathrm{~m}$ from the interaction point (IP). Near-beam proton taggers will be installed by TOTEM at $\pm 147 \mathrm{~m}$ and $\pm 220 \mathrm{~m}$ from the IP. The kinematic coverage of the combined CMS and TOTEM apparatus is unprecedented at a hadron collider. The CMS and TOTEM collaborations have described the considerable physics potential of joint data taking in a report to the LHCC [1]. Further near-beam proton taggers in combination with very fast timing detectors to be installed at $\pm 420 \mathrm{~m}$ from the IP (FP420) are in the proposal stage in CMS. FP420 would give access to possible discovery processes in forward physics at the LHC [2].

\section{Physics with forward detectors}

The CMS HF, CASTOR and ZDC calorimeters [3] and the TOTEM T1 and T2 telescopes [4] are particulatly suited for QCD studies at very low values of Bjorken- $x$, which have the potential of differentiating between DGLAP-type and BFKL-like parton evolution and of identifying effects of parton saturation. Measurements at the HERA ep collider have explored low- $x_{B j}$ dynamics down to values of a few $10^{-5}$. At the LHC the minimum accessible $x$ decreases by a factor $\sim 10$ for each 2 units of rapidity. A process with a hard scale of $Q \sim 10 \mathrm{GeV}$ and within the acceptance of T2/CASTOR $(\eta=6)$ can occur at $x$ values as low as $10^{-6}$.

An example is Drell-Yan production of $e^{+} e^{-}$pairs, $q q \rightarrow \gamma^{\star} \rightarrow e^{+} e^{-}$, which probes primarily the quark content of the proton. At values sufficiently low in $x$ non-linear QCD effects of parton recombination and saturation within the proton are expected to set in, taming the rapid rise of the proton gluon density observed by HERA and preserving unitarity. Generator-level studies [1] indicate that saturation effects may manifest themselves as production cross sections for DrellYan electrons within the T2/CASTOR acceptance that are sizably different from those predicted in models without saturation.

Another example of a low- $x$ process is production of forward jets, observable in HF or CASTOR. The CMS HF calorimeter offers excellent jet reconstruction capabilities. Forward jets in HF reach a $p_{T}$ resolution of $\sim 19 \%$ at $20 \mathrm{GeV} / \mathrm{c}$ and $\sim 10 \%$ at $100 \mathrm{GeV} / \mathrm{c}$ [5]. Particularly sensitive 
to BFKL-like QCD evolution dynamics are dijets with large rapidity separation, which enhances the available phase space for BFKL-like parton radiation between the jets. Generator-level studies for these so-called Muller-Navelet-type dijets with $\Delta \eta$ up to 9 are discussed in [6]. Likewise dijets separated by a large rapidity gap are of interest since they indicate a process in which no color flow occurs in the hard scatter but where, contrary to the traditional picture of soft Pomeron exchange, also a high transverse momentum transfer occurs across the gap.

Another area where the CMS forward calorimeters and TOTEM telescopes are essential tools is Monte Carlo tuning. The hard scatter in hadron-hadron collisions takes place in a dynamic environment, refered to as the "underlying event" (UE), where additional soft or hard interactions between the partons and initial and final state radiation occur. The effect of the UE can only be described by means of tuning Monte Carlo multiplicities and energy flow predictions to data. As shown in [7], the forward detectors are sensitive to features of the UE that central detector information alone cannot constrain. High uncertainties are also present in modelling the interaction of primary cosmic rays of $\mathrm{PeV}$ energy with the atmosphere. Their rate of occurance per year is too low for reliable quantitative analysis. The center-of-mass energy in $p p$ collisions at the LHC corresponds to $100 \mathrm{PeV}$ energy in a fixed target collision. As discussed in [1], measurements of energy and particle flow with T2/CASTOR and ZDC will be able to constrain significantly the Monte Carlo models currently used in the cosmic rays community.

\section{Physics with a veto on the forward detetcors}

Events of the type $p p \rightarrow p X p$ or $p p \rightarrow X p$, where no color exchange takes place between the proton(s) and the system $X$, can be caused by $\gamma$ exchange, or by diffractive interactions. In both cases, the absence of color flow between the proton(s) and the system $X$ results in a large gap in the rapidity distribution of the hadronic final state. In the following, we discuss, for several exemplary processes, how the forward detectors can be used to detect such hadronic final states.

Diffraction can occur with a hard scale. In that case perturbative QCD (pQCD) allows the cross sections for these processes to be factorized into that one of the hard scatter and a diffractive particle distribution function (dPDF). In diffractive hadron-hadron scattering, rescattering between spectator particles breaks the factorization. The so-called rapidity gap survival probability, $\langle|\mathcal{F}|\rangle$, quantifies this effect [8] and can be measured by means of the ratio of diffractive to inclusive processes with the same hard scale. At the Tevatron, the ratio is found to be $\mathscr{O}(1 \%)$ [9]. Theoretical expectations for $<\left|S^{2}\right|>$ the LHC vary from a fraction of a percent to as much as $\mathscr{O}(25 \%)$ [10].

In hard single diffraction (SD) at LHC energies, the complication arises that the rapidity gap is generally boosted to large values of rapidity. A gap within the acceptance of the HF and CASTOR calorimeters limits the values of $\xi$, the fractional momentum loss of the diffractively scattered proton, to $\xi<0.01$. Two processes have been studied in detail [11, 12], SD dijet production, $p p \rightarrow p j j X$, sensitive to the dPDF gluon content, and SD $W$ production, $p p \rightarrow p W X(W \rightarrow \mu v)$, sensitive to the dPDF quark content. Both studies assume single-interaction data, i.e. absence of event pile-up. The event selection is based on the two dimensional distributions of towers with above-noise activity on the gap side, a. in HF versus CASTOR, or b. in a high- $\boldsymbol{\eta}$ slice versus a low- $\eta$ slice of HF towers. The gap side is defined as the side with the lower hadronic activity, a definition that can be equally applied to non-diffractive events. 
After event selection, the two-dimensional multiplicity distributions on the selected gap side show clearly visible differences between the Pyhtia-only prediction (no hard SD) and the Pythia plus Pomwig (hard SD) one. Assuming $\left\langle\left|S^{2}\right|>\approx 5 \%\right.$ in Pomwig, a particularly clear excess is present in the $[0,0]$ bins. For SD $W$ production, $\mathscr{O}(100)$ events per $100 \mathrm{pb}^{-1}$ are expected in $[\mathrm{n}(\mathrm{CASTOR}), \mathrm{n}(\mathrm{HF})]=[0,0]$, with a ratio of SD to non-diffractive events of $\sim 20$. Even more favorable is the situation for SD dijet production, where $\mathscr{O}(300)$ events per $10 \mathrm{pb}^{-1}$ are expected, with a ratio of $\sim 30$. Dissociation of the diffractively scattered proton would lead to a signal enhancement in the $[0,0]$ bin of $\sim 30 \%$ for both channels. The studies assume that CASTOR will be available only on one side in the first phase of CMS data taking. A second CASTOR in the opposite hemisphere and the use of T1, T2 will improve the observable excess further. A method to establish that the observed population of the $[0,0]$ bins is indeed indicative of the presence of $\mathrm{SD}$ events in the data is described in [12]. The method is based on the observation that the size of the SD signal in the $[0,0]$ bins can be controlled in a predictable way when the cuts for enhancing the SD signal are modified. Observation of signals in the $[0,0]$ bins of the size given above would already exclude values of $\left\langle\left|S^{2}\right|>\right.$ at the low end of the spectrum of theoretical predictions.

Exclusive dimuon and dielectron production with no significant additional activity in the CMS detector occurs with high cross section in $\gamma$-mediated processes at the LHC, either as the pure QED process $\gamma \gamma \rightarrow l l$ or in $\Upsilon$ photoproduction. The event selection in both cases [13] is based on requiring that outside of the two leptons, no other significant activity is visible within the central CMS detector, neither in the calorimeter nor in the tracking system.

For the pure QED process, in $100 \mathrm{pb}^{-1}$ of single interaction data, $\mathscr{O}(700)$ events in the dimuon channels and $\mathscr{O}(70)$ in the dielectron channel can be selected. Events in which one of the protons dissociates are the dominant source of background and are comparable in statistics to the signal. This background can be significantly reduced by means of a veto on activity in CASTOR and ZDC, by $2 / 3$ in a configuration with a ZDC on each side and a CASTOR on only one side of the IP. The theoretically very precisely known cross section of this (almost) pure QED process is an ideal calibration channel. With $100 \mathrm{pb}^{-1}$ of single-interaction data, an absolute luminosity calibration with $\mathscr{O}(5 \%)$ precision is feasible. Futhermore, exclusive dimuon production is an ideal alignment channel with high statistics for the proposed proton taggers at $420 \mathrm{~m}$ from the IP.

Upsilon photoproduction can constrain QCD models of diffraction. Assuming the STARLIGHT Monte Carlo cross section prediction, the $1 \mathrm{~S}, 2 \mathrm{~S}$ and $3 \mathrm{~S}$ resonances will be clearly visible in $100 \mathrm{pb}^{-1}$ of single interaction data [13]. By means of $p_{T}^{2}(\Upsilon)$ as estimator of the transfered fourmomentum squared, $t$, at the proton vertex, it might be possible to measure the $t$ dependence of the cross section. This dependence is sensitive to the two-dimensional gluon distribution of the proton and would give access to the generalized parton distribution function (GPD) of the proton.

\section{Physics with near-beam proton taggers}

The LHC beamline with its magnets is essentially a spectrometer in which protons slightly off the beam momentum are bent sufficiently to be detectable by means of detectors inserted into the beam-pipe. At high luminosity at the LHC, proton tagging is the only means of detecting diffractive and $\gamma$ mediated processes because areas of low or no hadronic activity in the detector are filled in by particles from overlaid pile-up events. 
The TOTEM proton taggers at $\pm 220 \mathrm{~m}$ at nominal LHC optics have acceptance for scattered protons from the IP for $0.02<\xi<0.2$. Smaller values of $\xi, 0.002<\xi<0.02$, can be achieved with proton taggers at $\pm 420 \mathrm{~m}$. The FP420 proposal [2] foresees employing 3-D Silicon, an extremely radiation hard novel Silicon technology, for the proton taggers, and additional fast timing Cherenkov detectors for the rejection of protons from pile-up events. The proposal is currently under consideration in CMS. If approved, installation could proceed in 2010, after the LHC start-up.

Forward proton tagging capabilities enhance the physics potential of CMS. They would render possible a precise measurement of the mass and quantum numbers of the Higgs boson should it be discovered by traditional searches. They also augment the CMS discovery reach for Higgs production in the minimal supersymmetric extension (MSSM) of the Standard Model (SM) and for physics beyond the SM in $\gamma p$ and $\gamma \gamma$ interactions. A case in point is the central exclusive production (CEP) process [14], $p p \rightarrow p+\phi+p$, where the plus sign denotes the absence of hadronic activity between the outgoing protons, which survive the interaction intact, and the state $\phi$. The final state consists solely of the scattered protons, which may be detected in the forward proton taggers, and the decay products of $\phi$ which can be detected in the central CMS detector. Selection rules force the produced state $\phi$ to have $J^{C P}=n^{++}$with $n=0,2, \ldots$ This process offers hence an experimentally very clean laboratory for the discovery of any particle with these quantum numbers that couples strongly to gluons. Additional advantages are the possibility to determine the mass of the state $\phi$ with excellent resolution from the scattered protons alone, independent of its decay products, and the possibility, unique at the LHC, to determine the quantum numbers of $\phi$ directly from the azimuthal asymmetry between the scattered protons. Forward proton tagging will also give access to a rich QCD program on hard diffraction at high luminosities and to precision studies of $\gamma p$ and $\gamma \gamma$ interactions at center-of-mass energies never reached before.

\section{References}

[1] CMS collab., TOTEM collab., CERN-LHCC-2006-039/G-124 (2006)

[2] FP420 collab., arXiv:0806.0302, [hep-ex]

[3] CMS collab., CERN-LHCC-2006-001 (2006)

[4] TOTEM collab., CERN-LHCC-2004-002 (2004)

[5] CMS collab., CMS PAS FWD-08-001 (2008)

[6] S. Cerci, D. D’Enterria, CMS CR-2008/097 (2008)

[7] K. Borras, Proceedings of DIS 2007

[8] J.D.Bjorken, Phys.Rev.D 47 (1993) 101; A.B. Kaidalov et al., Eur.Phys.J.C21 (2001) 521

[9] CDF collab., Phys.Rev.Lett. 78 (1997) 2698; D0 collab., Phys.Lett.B 574 (2003) 169

[10] V. A. Khoze, A. D. Martin, M. G. Ryskin, arXiv:0802.0177 [hep-ph]; M. B. G. Ducati, M. M. Machado, M. V. T. Machado, arXiv:0706.3468 [hep-ph]

[11] CMS collab., CMS PAS DIF-07-002 (2008)

[12] CMS collab., CMS PAS FWD-08-002 (2008)

[13] CMS collab., CMS PAS DIF-07-001 (2008)

[14] V. A. Khoze, A. D. Martin, M. G. Ryskin., Eur.Phys.J C19 (2001) 477, erratum C20 (2001) 599 